MATHEMATICS OF COMPUTATION

Volume 70, Number 233 , Pages $269-279$

S $0025-5718(00) 01198-4$

Article electronically published on March 3, 2000

\title{
CONSTRUCTING FULLY SYMMETRIC CUBATURE FORMULAE FOR THE SPHERE
}

\author{
SANGWOO HEO AND YUAN XU
}

\begin{abstract}
We construct symmetric cubature formulae of degrees in the 1339 range for the surface measure on the unit sphere. We exploit a recently published correspondence between cubature formulae on the sphere and on the triangle. Specifically, a fully symmetric cubature formula for the surface measure on the unit sphere corresponds to a symmetric cubature formula for the triangle with weight function $\left(u_{1} u_{2} u_{3}\right)^{-1 / 2}$, where $u_{1}, u_{2}$, and $u_{3}$ are homogeneous coordinates.
\end{abstract}

\section{INTRODUCTION}

In this paper we construct symmetric cubature formulae on the surface of the sphere $S^{2}$ in $\mathbb{R}^{3}$ by using a correspondence between cubature formulae on the sphere and on the simplex established in [23]. Throughout this paper we denote by $\Pi_{n}^{d}$ the space of polynomials of degree at most $n$ in $d$ variables $(d=2$ or 3$)$, and we denote by $T$ the triangle with vertices at $(0,0),(1,0)$ and $(0,1)$. Let $W\left(y_{1}^{2}, y_{2}^{2}, y_{3}^{2}\right)$ be a weight function defined on $\mathbb{R}^{3}$, normalized so that $\int_{S^{2}} W\left(y_{1}^{2}, y_{2}^{2}, y_{3}^{2}\right) d \omega=1$. Associated with $W$, we define a weight function $W_{T}$ on the triangle $T$ by

$$
W_{T}\left(u_{1}, u_{2}\right)=2 W\left(u_{1}, u_{2}, 1-u_{1}-u_{2}\right) / \sqrt{u_{1} u_{2}\left(1-u_{1}-u_{2}\right)}, \quad\left(u_{1}, u_{2}\right) \in T .
$$

Then the correspondence between cubature formulae on $S^{2}$ and on $T$ states that

Theorem 1.1. Let $W$ and $W_{T}$ be defined as above. Suppose that there is a cubature formula of degree $M$ on $T$ given by

$$
\int_{T} f\left(u_{1}, u_{2}\right) W_{T}\left(u_{1}, u_{2}\right) d u_{1} d u_{2}=\sum_{k=1}^{N} \lambda_{k} f\left(u_{k, 1}, u_{k, 2}\right), \quad f \in \Pi_{M}^{2},
$$

whose $N$ nodes lie on the triangle $T$. Then there is a cubature formula of degree $2 M+1$ on the unit sphere $S^{2}$,

$$
\begin{aligned}
& \int_{S^{2}} g\left(y_{1}, y_{2}, y_{3}\right) W\left(y_{1}^{2}, y_{2}^{2}, y_{3}^{2}\right) d \omega \\
& \quad=\sum_{k=1}^{N} \lambda_{k} \sum_{\varepsilon_{i}= \pm 1} g\left(\varepsilon_{1} v_{k, 1}, \varepsilon_{2} v_{k, 2}, \varepsilon_{3} v_{k, 3}\right) / 2^{a_{k}}, \quad g \in \Pi_{2 M+1}^{3},
\end{aligned}
$$

Received by the editor July 8, 1997 and, in revised form, February 6, 1998, July 14, 1998, and January 12, 1999.

2000 Mathematics Subject Classification. Primary 65D32, 41A55, 41A63.

Key words and phrases. Cubature formulae, on the unit sphere, on the triangle, symmetric formula on a triangle, octahedral symmetry.

Supported by the National Science Foundation under Grants DMS-9500532 and 9802265.

(C)2000 American Mathematical Society 
where $a_{k}$ is the number of nonzero elements among $v_{k, 1}, v_{k, 2}$ and $v_{k, 3}$, and the nodes $\left(v_{k, 1}, v_{k, 2}, v_{k, 3}\right) \in S^{2}$ are defined in terms of $\left(u_{k, 1}, u_{k, 2}\right)$ by

$$
\left(v_{k, 1}, v_{k, 2}, v_{k, 3}\right)=\left(\sqrt{u_{k, 1}}, \sqrt{u_{k, 2}}, \sqrt{1-u_{k, 1}-u_{k, 2}}\right) .
$$

On the other hand, if there exists a cubature formula of degree $2 M+1$ on $S^{2}$ in the form of (1.3), then there is a cubature formula of degree $M$ on the simplex $T$ in the form of $(1.2)$ whose nodes $\left(u_{k, 1}, u_{k, 2}\right) \in T$ are defined by $\left(u_{k, 1}, u_{k, 2}\right)=\left(v_{k, 1}^{2}, v_{k, 2}^{2}\right)$.

The formula (1.3) is invariant under the change of signs, or invariant under the group $\mathbb{Z}_{2} \times \mathbb{Z}_{2} \times \mathbb{Z}_{2}$. The theorem establishes the equivalence between (1.2) and (1.3). In [23] this theorem is proved more generally for formulae on the sphere $S^{d}$ and the simplex $\Sigma^{d}$ for all $d$. For $d=2$ we have used the more customary notation $T$ for the simplex $\Sigma^{2}$. When $W(\mathbf{y})=1 / 4 \pi$ is the reciprocal of the surface area of $S^{2}$, the corresponding weight function on $T$ is the multiple of the weight function $\left(u_{1} u_{2}\left(1-u_{2}-u_{3}\right)\right)^{-1 / 2}$, which we will denote by $W_{0}$; that is,

$$
W_{0}\left(u_{1}, u_{2}\right)=\left(u_{1} u_{2}\left(1-u_{1}-u_{2}\right)\right)^{-1 / 2} / 2 \pi, \quad\left(u_{1}, u_{2}\right) \in T .
$$

In the following section we adopt a method of Lyness and Jespersen [15] to construct symmetric cubature formulae on $T$, which are formulae that are invariant under the symmetric group of the triangle, and use Theorem 1.1 to generate cubature formulae on the sphere. When the formula (1.2) on $T$ is symmetric, the corresponding formula (1.3) in Theorem 1.1 is invariant under the octahedral group, which is the symmetric group of the unit cube $\{ \pm 1, \pm 1, \pm 1\}$ in $\mathbb{R}^{3}$. In this case, the formula (1.3) is of the form

$$
\begin{aligned}
& \int_{S^{2}} g\left(y_{1}, y_{2}, y_{3}\right) W\left(y_{1}^{2}, y_{2}^{2}, y_{3}^{2}\right) d \omega \\
& \quad=\sum_{k=1}^{N} \mu_{k} \sum_{\sigma} \sum_{\varepsilon_{i}= \pm 1} g\left(\varepsilon_{1} v_{k, \sigma_{1}}, \varepsilon_{2} v_{k, \sigma_{2}}, \varepsilon_{3} v_{k, \sigma_{3}}\right), \quad g \in \Pi_{2 M+1}^{3},
\end{aligned}
$$

where the second sum is taken over all permutations of $\sigma=\left(\sigma_{1}, \sigma_{2}, \sigma_{3}\right)$, and $\mu_{k}=$ $\lambda_{k} /\left(b_{k} \cdot 2^{a_{k}}\right)$ with $b_{k}=\sum_{\sigma} 1$ for the corresponding point. Formulae of this type have been constructed by Lebedev [10]-[13. They are called fully symmetric in [20] and [7, and have been studied for $S^{d}$ in [8], which contains another correspondence between fully symmetric formulae on $S^{d}$ and cubature formulae on $\Sigma^{d}$, namely, a correspondence between the consistent rule structures on these two regions.

Numerical integration on the sphere has attracted a lot of attention; we refer to [1, 2], 4], 10]-[13, [17- 20] and the references there. Most formulae have been constructed by making use of symmetry to reduce the number of moment equations that have to be solved (see, for example, Sobolev [19] and McLaren [17). The fundamental result of Sobolev states that a cubature formula invariant under a finite group is exact for all polynomials in a subspace $\mathcal{P}$ if, and only if, it is exact for all polynomials in $\mathcal{P}$ that are invariant under the same group. The group that has been employed previously in this context is the octahedral group; Lebedev constructed in [10]-13] cubature formulae of degree up to 59, many of which have the smallest number of nodes among all formulae that are known. Working with symmetric cubature formulae on $T$, we are able to find many formulae on $S^{2}$ of a structure that Lebedev did not consider (see the following section). There are also formulae that are invariant under the icosahedral group, which have, however, no corresponding formulae on the triangle, since they are not symmetric under 
$\mathbb{Z}_{2} \times \mathbb{Z}_{2} \times \mathbb{Z}_{2}$ in the first place. We refer to [4, [18, [20] and the references there for other papers that deal with cubature formulae on the sphere; see also [1], in which formulae are constructed making use of symmetry and a Taylor expansion formula.

\section{Symmetric FORMUlaE ON $T$ \\ AND FULLY SYMMETRIC FORMULAE ON $S^{2}$}

In this section, we consider symmetric formulae with respect to the weight function $W_{0}$ on $T$, which correspond to cubature formulae with octahedral symmetry on $S^{2}$. In the first part of the section we present a method of constructing symmetric formulae given by Lyness and Jespersen in [15]. Our findings of cubature formulae are discussed in subsection 2.2, and we discuss the numerical computation in subsection 2.3 .

2.1. Symmetric formulae on the triangle. Instead of $T$, Lyness and Jespersen used the equilateral triangle

$$
\triangle=\{(x, y): x \leq 1 / 2, \quad \sqrt{3} y-x \leq 1, \quad-\sqrt{3} y-x \leq 1\},
$$

whose symmetric group $\mathcal{S}_{3}(\triangle)$ is generated by a rotation through an angle $2 \pi / 3$ and a reflection about the $x$-axis. The triangle $T$ can be transformed into $\triangle$ by the affine transformation

$$
\begin{gathered}
\varphi:\left(x_{1}, y_{1}\right) \in T \mapsto(x, y) \in \triangle, \\
x=3\left(x_{1}+x_{2}\right) / 2-1, \quad y=\sqrt{3}\left(x_{2}-x_{1}\right) / 2 .
\end{gathered}
$$

It is easy to see that invariance is preserved under $\varphi$; in particular, if a function $f$ defined on $\triangle$ is invariant under $\mathcal{S}_{3}(\triangle)$, then the function $f \circ \varphi$ defined on $T$ is invariant under $\mathcal{S}_{3}(T)$. The weight function $W_{0}$ on $T$ becomes

$$
W_{\triangle}^{0}(x, y)=3^{-3 / 2}\left((1+x)^{2}-3 y^{2}\right)^{-1 / 2}(1-2 x)^{-1 / 2} /(2 \pi) .
$$

A basis for the class of $\mathcal{S}_{3}(\triangle)$-invariant polynomials of degree at most $n$ can be written down in terms of the polar coordinates $x=r \cos \theta$ and $y=r \sin \theta$ as follows:

$$
r^{2 i}\left(r^{3} \cos 3 \theta\right)^{j}, \quad 0 \leq 2 i+3 j \leq n .
$$

Moreover, working with functions $g(r, \theta)=f(r \cos \theta, r \sin \theta)$ in polar coordinates, a basic invariant cubature formula takes the form

$$
Q(r, \theta) g=\frac{1}{6} \sum_{j=1}^{3}\left\{g\left(r, \theta+\frac{2 \pi j}{3}\right)+g\left(r,-\theta+\frac{2 \pi j}{3}\right)\right\},
$$

which is just a sum over the $\mathcal{S}_{3}(\triangle)$-orbit of the point $(r, \theta)$. Because of the invariance of $Q(r, \theta)$, we assume that $r$ can take negative values and $0 \leq \theta<\pi / 3$. Three distinct types of orbits occur according to whether $r=0$ (center of triangle); $r \neq 0$, $\cos 3 \theta=1$ (median of triangle); or $r \neq 0, \cos 3 \theta \neq 1$. These three types are denoted as holistic type 0 , holistic type 1 and holistic type 2 , whose corresponding $Q(r, \theta) g$ requires 1,3 , or 6 function evaluations, respectively. Let $n_{i}$ denote the number of orbits of type $i$ in a symmetric cubature formula. The general symmetric cubature formula takes the form

$$
Q(g)=n_{0} \lambda_{0} g(0,0)+\sum_{i=1}^{n_{1}} \lambda_{i} Q\left(r_{i}, 0\right) g+\sum_{i=n_{1}+1}^{n_{1}+n_{2}} \lambda_{i} Q\left(r_{i}, \theta_{i}\right) g .
$$


The number of nodes of this formula, denoted by $\mu(Q)$, is $\mu(Q)=n_{0}+3 n_{1}+6 n_{2}$. It is shown in [15] that the cubature formula $Q(g)$ is of degree $M$ if its nodes and weights satisfy the following system of equations:

$$
\begin{gathered}
\lambda_{0}+\sum_{i=1}^{n_{1}} \lambda_{i}+\sum_{i=n_{1}+1}^{n_{1}+n_{2}} \lambda_{i}=v_{0,0}, \\
\sum_{i=1}^{n_{1}} \lambda_{i} r_{i}^{j}+\sum_{i=n_{1}+1}^{n_{1}+n_{2}} \lambda_{i} r_{i}^{j} \cos 3 k \theta_{i}=v_{j, 3 k}, \quad 2 \leq j \leq M, k=k_{0}, \\
\sum_{2 i=n_{1}+1}^{n_{1}+n_{2}} \lambda_{i} r_{i}^{j}\left(\cos 3 k_{0} \theta_{i}-\cos 3 k \theta_{i}\right)=v_{j, 3 k}, \quad j=6, \text { or } 8 \leq j \leq M, \\
6 \leq 3 k \leq j, j+k \text { even, }
\end{gathered}
$$

where $k_{0}=0$ if $j$ is even and $k_{0}=1$ if $j$ is odd, and the numbers $v_{j, 3 k}$ are defined by

$$
v_{j, 3 k}=\int_{\triangle} r^{j+1} \cos 3 k \theta W_{\triangle}^{0}(r \cos \theta, r \sin \theta) d r d \theta,
$$

where the integral is over the region defined by $(r \cos \theta, r \sin \theta) \in \triangle$. For each $M$, the system contains

$$
E(M)=\left[\left(M^{2}+6 M+12\right) / 12\right]
$$

equations, where $[x]$ denote the greatest integer less than or equal to $x$.

It is often useful to construct cubature formulae that have some nodes on the edges or at the vertices of the triangle. To describe such a formula, we use the cytolic types (orbits) introduced in [15]. The holistic type $1(r \neq 0, \cos 3 \theta=1)$ orbits may be sub-partitioned into cytolic type 1: $r=-1$ (vertices), cytolic type 2 : $r=1 / 2$ (mid point of edges), and cytolic type $3:-1<r<1 / 2$. The holistic type 2 $(r \neq 0, \cos 3 \theta \neq 0)$ orbits may be sub-partitioned into cytolic type $4: r \cos \theta=1 / 2$ (nodes on the edges but not at mid point of the edges nor at vertices), and cytolic type 5: $r \cos \theta \neq 1 / 2$ (interior points). From here on we treat only cytolic rules. The structure of a cytolic rule is denoted by $\left[m_{0} ; m_{1}, m_{2}, m_{3} ; m_{4}, m_{5}\right]$, where $m_{j}$ denotes the number of components of cytolic type $j$ contained in the rule. In particular, we have $n_{0}=m_{0}, n_{1}=m_{1}+m_{2}+m_{3}$, and $n_{2}=m_{4}+m_{5}$. We note that $m_{0}, m_{1}$ and $m_{2}$ can take only values either 0 or 1 .

According to Theorem 1.1, a cytolic cubature formula of structure $\left[m_{0} ; m_{1}, m_{2}\right.$, $m_{3} ; m_{4}, m_{5}$ ] leads to a cubature formula on $S^{2}$ whose number of nodes is equal to

$$
N\left(S^{2}\right)=8 m_{0}+6 m_{1}+12 m_{2}+24 m_{3}+24 m_{4}+48 m_{5} .
$$

The nonlinear system of equations (2.4) remains in the same form for the cytolic type formulae; we only need to assign proper values of certain $r_{i}$ and $\theta_{i}$ according to the given type. To form the nonlinear system of equations (2.4), we choose $m_{0}$ and $m_{i}$ so that the number of parameters matches the number of equations. For the structure $\left[m_{0} ; m_{1}, m_{2}, m_{3} ; m_{4}, m_{5}\right]$, this means

$$
m_{0}+m_{1}+m_{2}+2 m_{3}+2 m_{4}+3 m_{5}=\left[\left(M^{2}+6 M+12\right) / 12\right],
$$

where $M$, as before, is the degree of the cubature formula. For each fixed $M$ there may be a number of integer solutions to the above equation, leading to different types of cubature formulae. In this regard, the consistency conditions are very 
useful. Following the argument in [15] for the holistic type, the conditions for the cytolic type are

$$
\begin{aligned}
2 m_{4}+3 m_{5} & \geq E(M-6), \\
m_{1}+m_{2}+2\left(m_{3}+m_{4}\right)+3 m_{5} & \geq E(M)-1, \\
m_{0}+m_{1}+m_{2}+2\left(m_{3}+m_{4}\right)+3 m_{5} & \geq E(M) .
\end{aligned}
$$

They are also included in the conditions found in [7] for the $d$-dimensional simplex. These conditions ensure that, within any subsystem of (2.4), the number of unknown parameters cannot be less than the number of equations. Another useful restriction is as follows.

Theorem 2.1. A formula of degree $M$ is of structure $\left[m_{0} ; m_{1}, m_{2}, m_{3} ; m_{4}, m_{5}\right]$ only if

$$
m_{5}> \begin{cases}(M-9) / 4, & \text { if } m_{4} \neq 0 \text { and } M \geq 9, \\ (M-6) / 4, & \text { if } m_{4}=0 \text { and } M \geq 6, \\ (M-3) / 4, & \text { if } m_{3}=0 \text { and } M \geq 3 .\end{cases}
$$

Proof. Let $\ell_{i}, i=1,2,3$, be the linear polynomials such that $\ell_{i}=0$ give the equations of the sides, and we choose the sign so that the $\ell_{i}$ are nonnegative on $\triangle$. Let $h_{i}, i=1,2,3$, be the linear polynomials such that $h_{i}=0$ give the equations of the medians of $\triangle$. Furthermore, let $\mathbf{x}_{i}=\left(r_{i} \cos \theta_{i}, r_{i} \sin \theta_{i}\right)$ be points of type 3 , and let $g_{i}, i=1,2, \ldots, m_{5}$, be the quadratic polynomials so that $g_{i}=0$ gives the equation of the circle that has center at origin and radius $r_{i}$. If $m_{4} \neq 0$, then the polynomial

$$
\ell_{1} \ell_{2} \ell_{3} h_{1}^{2} h_{2}^{2} h_{3}^{2} g_{1}^{2} \ldots g_{m_{5}}^{2}
$$

will vanish on all nodes of the formula. Since the polynomial is positive on $\triangle$, its degree has to be bigger than the degree of the cubature formula, which leads to the desired inequality. If $m_{4}=0$, then the factors $\ell_{1} \ell_{2} \ell_{3}$ can be dropped from the polynomial, leading to the desired inequality in this case. If $m_{3}=0$, then $h_{1}^{2} h_{2}^{2} h_{3}^{2}$ can be dropped from the polynomial.

This theorem and its proof are extensions of the result in [15, p. 26], which deals with the cases of $M=5,6,9$. There are other conditions that can be derived this way; for example, if both $m_{3}$ and $m_{4}$ are zero, then $m_{5}>M / 4$. For fixed $M$, it is possible to identify all possible integer solutions of (2.7) which also satisfy the restrictions (2.8) and (2.9); the number of the solutions, however, is still large even for moderate $M$. For more general conditions of this type, we refer to [7] and [16].

Particular choices of structure lead to a system (2.4) that may be split into subsystems, each having fewer independent variables: the smaller size of the subsystem makes them easier to solve. Such a split is possible since the third group of the equations in (2.4) does not contain $r_{i}$ and $\lambda_{i}$ for $i=1,2, \ldots, n_{1}$; and it occurs whenever $m_{4}$ and $m_{5}$ satisfy the equation

$$
2 m_{4}+3 m_{5}=E(M-6)=\left[\left(M^{2}-6 M+12\right) / 12\right],
$$

because the third group of equations contain $E(M-6)$ independent parameters. It is not hard to check that the integer solutions of the above equation exist for every $M \geq 7$, except $M=10$; hence, the splitting occurs for each $M \neq 10$. One important class of formulae that admits the splitting corresponds to the cubature formulae constructed by Lebedev in [10]-13] on $S^{2}$ with octahedral symmetry. Apart from 
a few lower degree cases, Lebedev considers the formulae on $S^{2}$ that correspond to the structures

$$
[1 ; 1,0,3 m ; m, m(m-1)] \quad \text { and } \quad\left[1 ; 1,1,3 m+1 ; m, m^{2}\right],
$$

which are of degree $6 m+2$ and $6 m+5$, respectively; and he has constructed formulae for $m=1,2,3,4$.

2.2. Fully symmetric cubature formulae on $S^{2}$. We have attempted to find symmetric cubature formulae of degree up to 20 on the triangle. Our strategy for choosing the structure $\left[m_{0} ; m_{1}, m_{2}, m_{3} ; m_{4}, m_{5}\right]$ is as follows. We search for types whose corresponding formulae on $S^{2}$ have fewer nodes. This means finding $m_{0}$ and $m_{i}$, which satisfy $(2.7),(2.8)$ and $(2.9)$, so that $N$ in (2.6) is minimal or close to minimal. To this end, we choose $m_{0}, m_{1}$ and $m_{2}$ with value one whenever possible, and then $m_{5}$ as small as possible. As a starting point, we choose $m_{4}$ and $m_{5}$ to satisfy (2.10), so that the system (2.4) is split into subsystems. There are some nonlinear systems for which we found no solution. For each $M \leq 20$, however, we found at least one cubature formula that has all of its nodes inside $\triangle$ and contains no negative weights; these correspond to cubature formulae on $S^{2}$ of degree up to 41. Note that, when such a formula for $\triangle$ has a node outside $\triangle$, Theorem 1.1 cannot be exploited to find a cubature formulae on $S^{2}$.

We report our findings as fully symmetric cubature formulae on $S^{2}$ and list the results in Table 2.1 below. Each formula is identified by its structure $\left[m_{0} ; m_{1}, m_{2}, m_{3}\right.$; $\left.m_{4}, m_{5}\right]$, and we give its number of nodes. If a formula has all positive weight, we write $P$ in the last column, otherwise, we write $N$.

The structures marked by $[\mathrm{S}]$ correspond to formulae in Stroud's book [20], structures marked by [L] correspond to formulae on $S^{2}$ found by Lebedev. The structures $[0 ; 1,0,2 ; 1,0]$ of degree 13 and $[1 ; 1,0,2 ; 2,0]$ of degree 17 have been constructed by Keast in 7, but the numerical values of the nodes and weights are not given there. All other formulae in the table appear to be new; in particular, these include formulae of degrees $21,31,33,37$ and 39 , where no formulae of the same degree have been published previously, and formulae of degrees 25 and 27 of quality P. Three formulae, of degree 47, 53 and 59, may be found in [12] and [13. Because the system of equations (2.4) is nonlinear, its solution need not be unique. In the cases of $[0 ; 0,0,3 ; 1,2]$ of degree 21 and $[0 ; 0,0,4 ; 1,10]$ of degree 39 , we found two solutions in each case, and we mark these cases by (2) in the table.

Numerical computation that leads to symmetric cubature formulae for the unit weight function is carried out in [15] for $M \leq 11$ and in [3] for $M \leq 20$. The equations (2.4) in the cases of the unit weight function and the weight function $W_{0}$ are of the same form, except that the moments are different, which only changes the right hand sides of the equations. Since the equations are nonlinear, formulae of the same type may possess different quality for different weight functions. For example, for the structure $[1 ; 0,0,6 ; 1,2]$ of degree 13 , we found a formula for $W_{0}$ with some negative weights, while the formula for the unit weight function has all positive weights. The most interesting case, however, is perhaps the structure $[1 ; 0,0,8 ; 1,7]$ of degree 19 , in which we found no solution for the weight function $W_{0}$, while a solution exists for the unit weight function (3]). This demonstrates once more the well-known phenomenon that the solutions of nonlinear systems may be sensitive to any changes in the value of any incidental constant. 
TABLE 2.1. Fully symmetric cubature formula on $S^{2}$

\begin{tabular}{|c|c|c|c|}
\hline Degrees & Structure & \# of Nodes & Quality \\
\hline \multirow[t]{2}{*}{3} & $0 ; 1,0,0 ; 0,0$ & 6 & $\mathrm{P}[\mathrm{S}]$ \\
\hline & $1 ; 0,0,0 ; 0,0$ & 8 & $\mathrm{P}[\mathrm{S}]$ \\
\hline 5 & $1 ; 1,0,0 ; 0,0$ & 14 & $\mathrm{P}[\mathrm{S}]$ \\
\hline \multirow[t]{2}{*}{7} & $1 ; 1,1,0 ; 0,0$ & 26 & $\mathrm{P}[\mathrm{S}]$ \\
\hline & $1 ; 0,0,1 ; 0,0$ & 32 & $\mathrm{~N}[\mathrm{~S}]$ \\
\hline 9 & $1: 1,0,0 ; 1,0$ & 38 & $\mathrm{P}\lceil\mathrm{L}]$ \\
\hline 11 & $1 ; 1,1,1 ; 0,0$ & 50 & $\mathrm{P}[\mathrm{S}]$ \\
\hline \multirow[t]{2}{*}{13} & $1 ; 1,1,1 ; 1,0$ & 74 & $\mathrm{~N}[\mathrm{~L}]$ \\
\hline & $0 ; 1,0,2 ; 1,0$ & 78 & $\mathrm{P}$ \\
\hline \multirow[t]{2}{*}{15} & $1 ; 1,0,2 ; 1,0$ & 86 & $\mathrm{P}[\mathrm{L}]$ \\
\hline & $0 ; 1,1,2 ; 1,0$ & 90 & $\mathrm{P}$ \\
\hline \multirow[t]{2}{*}{17} & $1 ; 1,0,3 ; 1,0$ & 110 & $\mathrm{P}[\mathrm{L}]$ \\
\hline & $1 ; 1,0,2 ; 2,0$ & 110 & $\mathrm{~N}$ \\
\hline \multirow[t]{3}{*}{19} & $1 ; 1,1,3 ; 0,1$ & 146 & $\mathrm{P}[\mathrm{L}]$ \\
\hline & $1 ; 1,1,2 ; 1,1$ & 146 & $\mathrm{P}$ \\
\hline & $1 ; 0,0,4 ; 0,1$ & 152 & $\mathrm{P}$ \\
\hline \multirow[t]{5}{*}{21} & $1 ; 1,1,3 ; 1,1$ & 170 & $\mathrm{~N}$ \\
\hline & $1 ; 1,1,2 ; 2,1$ & 170 & $\mathrm{~N}$ \\
\hline & $1 ; 0,0,3 ; 2,1$ & 176 & $\mathrm{~N}$ \\
\hline & $0 ; 0,0,3 ; 1,2$ & 192 & $\mathrm{P}(2)$ \\
\hline & $1 ; 0,0,2 ; 0,3$ & 200 & $\mathrm{P}$ \\
\hline \multirow[t]{4}{*}{23} & $1 ; 1,1,4 ; 1,1$ & 194 & $\mathrm{P}[\mathrm{L}]$ \\
\hline & $0 ; 1,0,4 ; 2,1$ & 198 & $\mathrm{P}$ \\
\hline & $1 ; 0,0,5 ; 1,1$ & 200 & $\mathrm{P}$ \\
\hline & $1 ; 0,0,4 ; 2,1$ & 200 & $\mathrm{~N}$ \\
\hline \multirow[t]{3}{*}{25} & $1 ; 1,0,5 ; 2,1$ & 230 & $\mathrm{~N}[\mathrm{~L}]$ \\
\hline & $1 ; 0,0,5 ; 1,2$ & 248 & $\mathrm{P}$ \\
\hline & $0 ; 0,0,5 ; 0,3$ & 264 & $\mathrm{P}$ \\
\hline \multirow[t]{4}{*}{27} & $1 ; 1,1,5 ; 1,2$ & 266 & $\mathrm{~N}[\mathrm{~L}]$ \\
\hline & $1 ; 0,0,6 ; 1,2$ & 272 & $\mathrm{~N}$ \\
\hline & $1 ; 1,0,5 ; 0,3$ & 278 & $\mathrm{~N}$ \\
\hline & $0 ; 0,0,5 ; 1,3$ & 288 & $\mathrm{P}$ \\
\hline \multirow[t]{2}{*}{29} & $1 ; 1,0,6 ; 2,2$ & 302 & $\mathrm{P}[\mathrm{L}]$ \\
\hline & $0 ; 0,0,6 ; 0,4$ & 336 & $\mathrm{P}$ \\
\hline 31 & $1 ; 0,0,4 ; 3,4$ & 368 & $\mathrm{P}$ \\
\hline$\overline{33}$ & $1 ; 0,0,6 ; 1,5$ & 416 & $\overline{\mathrm{P}}$ \\
\hline \multirow[t]{2}{*}{35} & $1 ; 1,1,7 ; 2,4$ & 434 & $\mathrm{P}[\mathrm{L}]$ \\
\hline & $1 ; 0,0,8 ; 2,4$ & 440 & $\mathrm{P}$ \\
\hline 37 & $1 ; 0,0,5 ; 1,8$ & 536 & $\mathrm{P}$ \\
\hline 39 & $0 ; 0,0,4 ; 1,10$ & 600 & $\mathrm{P}(2)$ \\
\hline$\overline{41}$ & $1 ; 1,0,9 ; 3,6$ & 590 & $\mathrm{P}[\mathrm{L}]$ \\
\hline
\end{tabular}


2.3. Remarks on numerical computation. The numerical computation was carried out on a DEC Alphastation 500 in double precision, using the DUNLSF Fortran subroutine in the IMSL Math/Library (Visual Numerics, Inc., 1994); however, moments $v_{j, 3 k}$ in (2.5) were computed exactly using Maple. The subroutine DUNLSF employs iterative techniques which require an initial estimate of the solution. For solving the nonlinear system (2.4), this means that we need to provide initial values for the weights $\lambda_{i}$ and for the parameters $r_{i}$ and $\theta_{i}$ that determine nodes. To determine the initial values, we have followed the strategy in [3] for solving the systems for the unit weight function. The node locations of the formulae for the weight function $W_{0}$ appear to be similar to those for the unit weight function: nodes are located closer to the edge of the triangle than the centroid, and are located closer to the median $\theta=\pi / 3$ than $\theta=0$. Our computation shows that whenever a formula of a given type has a solution, then even a rough initial estimate leads to the solution in reasonable computing time. For example, finding a formula of degree 19 needs less than 30 minutes.

For each formula of degree $M$, we compute the relative error and the absolute error of $\mathcal{I}(f)-\mathcal{I}_{n}(f)$ for all invariant polynomials $f$ of degree $\leq M$, where $\mathcal{I}(f)$ stands for the integral of $f$ with respect to $W_{0}$ on the triangle and $\mathcal{I}_{n}(f)$ stands for the numerical approximation (in principle but not in practice exact) to this quantity calculated using the cubature formula. We found that

$$
\sup \left\{\left|\mathcal{I}(f)-\mathcal{I}_{M}(f)\right| / \mathcal{I}(f): f \in \Pi_{M}^{G}\right\} \leq 0.5 \times 10^{-13}
$$

for formulae of degree up to 19, but it was ten times larger for the degree 20 . The numerical values of the parameters are given to 12 digits. The DUNLSF subroutine solves the nonlinear equations in the least square sense; that is, it finds the minimal solution of $\sum f_{i}^{2}(\mathbf{x})$, where $f_{i}=0$ are nonlinear equations. In our computation, equations in (2.4) involve high powers of polynomials which, however, seem not to be sensitive to perturbations; for example, for $M=20$, a perturbation in the 5 -th decimal place of our solution did not change the order of the relative error $10^{-12}$. For $M$ large, the accuracy of the solution found by the DUNLSF subroutine is limited by the machine accuracy. Because the computer we used has limited precision of 15 digits, we stopped at $M=21$.

\section{Final COMments}

We comment on some perspectives that are not covered in the present paper.

Remark 3.1. Theorem 1.1 establishes the connection between cubature formulae on $\Sigma^{d}$ and $\mathbb{Z}_{2} \times \cdots \times \mathbb{Z}_{2}$ symmetric cubature formulae on $S^{d}$. In [22] we also establish a connection between cubature formulae on the ball $B^{d}$ and on $S^{d}$, and that connection has been used to construct cubature formulae on $S^{2}$ in [5]. Together, these results yield a correspondence between cubature formulae on $\Sigma^{d}$ and $\mathbb{Z}_{2} \times \cdots \times \mathbb{Z}_{2}$ symmetric formulae on $B^{d}$. In particular, a cubature formula for the weight function $W_{0}$ on $T$ corresponds to a formula for the weight function $1 / \sqrt{1-x_{1}^{2}-x_{2}^{2}}$ on $B^{2}$. Thus, the results in Sections 2 and 3 also lead to $\mathbb{Z}_{2} \times \mathbb{Z}_{2} \times \mathbb{Z}_{2}$ symmetric formulae on $B^{2}$. On the other hand, those formulae constructed in [5] that are $\mathbb{Z}_{2} \times \mathbb{Z}_{2} \times \mathbb{Z}_{2}$ symmetric lead to formulae on $T$. However, not all formulae on $B^{2}$ in [5] are fully symmetric. In fact, the correspondence between formulae on $B^{d}$ and on $S^{d}$ is not restricted to $\mathbb{Z}_{2} \times \mathbb{Z}_{2} \times \mathbb{Z}_{2}$ symmetric formulae. 
Remark 3.2. The connection between cubature formulae on $S^{d}, \Sigma^{d}$ and $B^{d}$ may be exploited to treat a large class of weight functions. In particular, cubature formulae for the unit weight function on $T$ correspond to $\mathbb{Z}_{2} \times \mathbb{Z}_{2} \times \mathbb{Z}_{2}$ symmetric formulae for $\left|x_{1} x_{2} x_{3}\right| d \omega$ on $S^{2}$ and for a weight function $\left|x_{1} x_{2}\right|$ on $B^{2}$; and $\mathbb{Z}_{2} \times \mathbb{Z}_{2} \times \mathbb{Z}_{2}$ symmetric cubature formulae for the unit weight function on $B^{2}$ correspond to formulae for $\left(1 / \sqrt{x_{1} x_{2}}\right)$ on $T$. For examples of formulae for the unit weight function on these domains, see the references in [18, [20].

Remark 3.3. The connection between formulae on the three domains may also be applied in higher dimensions. Although a number of formulae of lower degrees have been constructed for the unit weight function in the literature (see [2], 44, [18, 20]), it may be of interest to construct formulae for the weight function $\left(u_{1} \cdots u_{d}\left(1-u_{1}-\ldots-u_{d}\right)\right)^{-1 / 2}$ on $\Sigma^{d}$ and use them to generate cubature formulae on $S^{d}$. To our knowledge, the calculation of symmetric cubature formulae for this weight function on $\Sigma^{d}$ for $d>2$ has not been undertaken previously, although the consistency conditions are available in 77 and $[16$. For the unit weight function, some symmetric formulae of lower degrees on $\Sigma^{d}$ have been constructed; see [2], 7], [18], 20] and the references given there.

\section{ACKNOWLEDGMENTS}

The authors thank the referees for their useful comments.

\section{Appendix}

We give the weights and nodes for the cubature formulae described in Section 2. The cubature formulae on $S^{2}$ are of the form (1.6) with $W(\mathbf{x})=1 / 4 \pi$. Because of the symmetry, for each weight $\mu_{k}$ we need to specify only one node $\left(v_{k, 1}, v_{k, 2}, v_{k, 3}\right)$. For a formula of structure $\left[m_{0} ; m_{1}, m_{2}, m_{3} ; m_{4}, m_{5}\right]$, the nodes corresponding to $m_{0}, m_{1}$ and $m_{2}$ are

$$
(\sqrt{1 / 3}, \sqrt{1 / 3}, \sqrt{1 / 3}), \quad(1,0,0), \quad(\sqrt{1 / 2}, \sqrt{1 / 2}, 0),
$$

involving eight, six, and twelve function values, respectively; the weights corresponding to these are denoted by $\mu_{0}, \mu_{1}$, and $\mu_{2}$, respectively. Note that some or all of $\mu_{0}, \mu_{1}, \mu_{2}$ could be zero, which means that the corresponding node does not appear in the formula.

For each formula we specify the value of nonzero $\mu_{i}, i=0,1,2$ first; those that are not given are understood as zero. We then list in tabular form the other nodes $\left(v_{i, 1}, v_{i, 2}, v_{i, 3}\right)$ and the corresponding weights $\mu_{i}$ starting with $i=3$ and following the ordering implied in the specified structure. That is, the weights corresponding to all nodes of type 3 are listed first, then all of those of type 4 followed by all those of type 5 . Here are the first three tables.

Degree 13: $[0 ; 1,0,2 ; 1,0] ; N=78 \quad \mu_{1}=0.013866592105$

\begin{tabular}{|c|c|c|c|c|}
\hline$i$ & $x_{i}$ & \multicolumn{1}{|c|}{$y_{i}$} & \multicolumn{1}{c|}{$z_{i}$} & $\mu_{i}$ \\
\hline 3 & 0.286640146767 & 0.914152532416 & 0.286640146767 & 0.013050931863 \\
4 & 0.659905001656 & 0.659905001656 & 0.359236381200 & 0.013206423223 \\
5 & 0.539490098706 & 0.841991943785 & 0.0 & 0.011942663555 \\
\hline
\end{tabular}


Degree 15: $[0 ; 1,1,2 ; 1,0] ; N=90$

$$
\mu_{1}=0.013191522874, \quad \mu_{2}=0.011024070845
$$

\begin{tabular}{|c|c|c|c|c|}
\hline$i$ & $x_{i}$ & $y_{i}$ & \multicolumn{1}{|c|}{$z_{i}$} & $\mu_{i}$ \\
\hline 3 & 0.337785899794 & 0.878522265967 & 0.337785899794 & 0.010538971114 \\
4 & 0.658511676782 & 0.658511676782 & 0.364314072036 & 0.011656960715 \\
5 & 0.399194381765 & 0.916866318264 & 0.0 & 0.010660818696 \\
\hline
\end{tabular}

$$
\begin{gathered}
\text { Degree 17: }[1 ; 1,0,2 ; 2,0] ; N=110 \\
\mu_{0}=0.009103396603, \quad \mu_{1}=-0.002664002664
\end{gathered}
$$

\begin{tabular}{|c|c|c|l|c|}
\hline$i$ & $x_{i}$ & \multicolumn{1}{|c|}{$y_{i}$} & \multicolumn{1}{c|}{$z_{i}$} & $\mu_{i}$ \\
\hline 3 & 0.357406744337 & 0.862856209461 & 0.357406744337 & 0.010777836655 \\
4 & 0.678598344546 & 0.678598344546 & 0.281084637715 & 0.009161945784 \\
5 & 0.542521185161 & 0.840042120165 & 0.0 & 0.009798544912 \\
6 & 0.222866509741 & 0.974848972321 & 0.0 & 0.009559874447 \\
\hline
\end{tabular}

Numerical values of the weights and abscissas of these and all other rules whose structures are given in Table 2.1 are listed in [6]. A machine-readable version of this technical memorandum is available on request from the authors.

\section{REFERENCES}

1. Z. P. Bažant and B. H. Oh, Efficient numerical integration on the surface of a sphere, Z. Angew. Math. Mech. 66 (1986), 37-49. MR 87f:68024

2. R. Cools and P. Rabinowitz, Monomial cubature rules since "Stroud": a compilation, J. Comp. Appl. Math. 48 (1993), 309-326. CMP 94:05

3. D. A. Dunavant, High degree efficient symmetrical Gaussian quadrature rules for the triangle, Internat. J. Numer. Methods Engrg. 21 (1985), 1129-1148. MR 86h:65029

4. H. Engels, Numerical quadrature and cubature, Academic Press, New York, 1980. MR 83g:65002

5. S. Heo and Y. Xu, Constructing cubature formulae for spheres and balls, J. Comp. Appl. Math. 12 (1999), 95-119. CMP 2000:06.

6. S. Heo and Y. Xu, Constructing cubature formulae for spheres and triangles, Technical Report, University of Oregon, 1998.

7. P. Keast, Cubature formulas for the surface of the sphere, J. Comp. Appl. Math. 17 (1987), 151-172. MR 88e:65027

8. P. Keast, Moderate-degree tetrahedral quadrature formulas, Comput. Methods Appl. Mech. Engrg. 55 (1986), 339-348. MR 87g:65035

9. P. Keast and J. C. Diaz, Fully symmetric integration formulas for the surface of the sphere in s dimensions, SIAM J. Numer. Anal. 20 (1983), 406-419. MR 84h:65025

10. V.I. Lebedev, Quadrature on a sphere, USSR Comp. Math. and Math. Phys. 16 (1976), 10-24. MR 55:11578

11. V.I. Lebedev, Spherical quadrature formulas exact to orders 25-29, Siberian Math. J. 18 (1977), 99-107. MR 56:7126

12. V.I. Lebedev, A quadrature formula for the sphere of 59th algebraic order of accuracy, Russian Acad. Sci. Dokl. Math. 50 (1995), 283-286. CMP 95:06

13. V.I. Lebedev and L. Skorokhodov, Quadrature formulas of orders 41,47 and 53 for the sphere, Russian Acad. Sci. Dokl. Math. 45 (1992), 587-592. MR 94b:65040

14. J.N. Lyness and R. Cools, A survey of numerical cubature over triangles, Mathematics of Computation 1943-1993: a half-century of computational mathematics (Vancouver, BC, 1993), Proc. Sympos. Appl. Math., 48, Amer. Math. Soc., Providence, RI, 1994, pp. 127150. MR 95j:65021

15. J.N. Lyness and D. Jespersen, Moderate degree symmetric quadrature rules for the triangle, J. Inst. Maths. Applics. 15 (1975), 19-32. MR 51:14536 
16. J. I. Maeztu and E. Sainz de la Maza, Consistent structures of invariant quadrature rules for the n-simplex, Math. Comp. 64 (1995), 1171-1192. MR 95j:65022

17. A. D. McLaren, Optimal numerical integration on a sphere, Math. Comp. 17 (1963), 361-383. MR 28:2635

18. I. P. Mysovskikh, Interpolatory cubature formulas, "Nauka", Moscow, 1981 (Russian). MR 83i:65025

19. S. L. Sobolev, Cubature formulas on the sphere invariant under finite groups of rotations, Sov. Math. Dokl. 3 (1962), 1307-1310. MR 25:4635

20. A. Stroud, Approximate calculation of multiple integrals, Prentice Hall, Englewood Cliffs, NJ, 1971. MR 48:5348

21. Y. Xu, On orthogonal polynomials in several variables, Special functions, $q$-series, and related topics, Fields Institute Communications, vol. 14, 1997, pp. 247 - 270. MR 99a:33009

22. Y. Xu, Orthogonal polynomials and cubature formulae on spheres and on balls, SIAM J. Math. Anal. 29 (1998), 779-793. MR 99j:33015

23. Y. Xu, Orthogonal polynomials and cubature formulae on spheres and on simplices, Methods Appl. Anal. 5 (1998), 169-184. CMP 98:16

Department of Mathematics, University of Oregon, Eugene, Oregon 97403-1222

E-mail address: yuan@math.uoregon.edu

Division of Science and Mathematics, University of Minnesota-Morris, Morris, MinNESOTA 56267

Current address: Department of Mathematics, University of Southern Indiana, Evansville, Indiana 47712

E-mail address: sheo@cda.mrs.umn.edu 